\title{
"Am stram gram » ou à la recherche des origines de la poésie
}

\section{Karolina Resztak}

\section{(2) OpenEdition}

\section{Journals}

Édition électronique

URL : http://journals.openedition.org/coma/589

DOI : $10.4000 /$ coma.589

ISSN : 2275-1742

\section{Éditeur}

Institut des textes \& manuscrits modernes (ITEM)

\section{Référence électronique}

Karolina Resztak, « «Am stram gram » ou à la recherche des origines de la poésie », Continents manuscrits [En ligne], 5 | 2015, mis en ligne le 15 octobre 2015, consulté le 21 décembre 2020. URL : http://journals.openedition.org/coma/589 ; DOI : https://doi.org/10.4000/coma.589

Ce document a été généré automatiquement le 21 décembre 2020.

\section{(2) $\odot \Theta \Theta$}

Continents manuscrits - Génétique des textes littéraires - Afrique, Caraîbe, dispora est mis à disposition selon les termes de la licence Creative Commons Attribution - Pas d'Utilisation

Commerciale - Pas de Modification 4.0 International. 


\title{
«Am stram gram » ou à la recherche des origines de la poésie
}

\author{
Karolina Resztak
}

«Il y a une porte que personne ne peut fermer.

La porte de l'imaginaire ${ }^{1}$ »

(Frankétienne)

1 Où commence donc la poésie? Dans le "Prière d'insérer" inédit retrouvé dans les archives de Formulaires ${ }^{2}$, M. Dib donne une réponse surprenante en proposant, en guise d'archétype du poème, une comptine d'enfant. Qui plus est, une comptine au texte peu intelligible et dont les origines semblent se perdre dans la nuit des temps ${ }^{3}$. Pérennisée grâce à sa musicalité, dépourvue de sens en français, cette comptine est reprise au fil des générations pour une grande liberté d'interprétations et pour son rythme captivant.

2 Force est de constater l'importance du rythme dans la poésie. M. Dib consacrait un temps considérable à travailler la prosodie de ses poèmes. Il était capable de reprendre un vers une quinzaine de fois et d'imaginer tous les jeux de mots possibles pour en affiner la musicalité.

Lui, le poète imaginait : tantôt il donnait, tantôt il brouillait le sens de son œuvre, laissant ses lecteurs imaginer les significations possibles. Il estimait qu'une fois publiés, ses romans et poèmes ne pouvaient exister qu'à travers l'imagination et l'interprétation des lecteurs et des critiques.

Les mots « imaginer » et « imaginaire » sont ici des mots-clés. Le talent d'imaginer, dans le sens de « créer », " concevoir » est au cœur de tout travail d'écriture, indépendamment du style auquel recourent les écrivains. Depuis le tournant d'Un été africain, M. Dib pratiquait ce que Michèle Coty a appelé l'«esthétique visionnaire [...] clivage et conjonction intentionnelle de la réalité et du rêve ${ }^{4}$ ». Selon l'écrivain lui-même, il en faut peu pour éveiller cette capacité puissante qui donne naissance à un poème : «[...] une certaine expression entrevue sur un visage, une certaine lumière tombant sur un mur, une certaine façon qu'ont les choses parfois de s'ordonner, et c'est la porte ouverte ${ }^{5}[. .$.$] ».$ Il suffit donc d'un rythme, d'une incantation, exactement comme dans une comptine. Le sens, le destinataire saura le donner grâce à ses propres capacités d'imagination. 

de M. Dib à l'époque où il travaillait sur Formulaires, le rêve et le poème sont très proches l'un de l'autre : "Ce sont de même des œuvres incohérentes dans leur déroulement, dans leur syntaxe, dans leur construction mais, comme les rêves justement, régies par une unité interne profonde $[. . .]^{7} »$. Le poème, comme le rêve, fait remonter à la surface de la conscience les pensées confinées au plus intime de l'être; il fait remonter ce qui tisse l'individu. En ce sens, le poème est l'expression de l'unité essentielle du moi.

5 Cette unité est bien visible dans tout le recueil. Elle est mise en relief dans ce "Prière d'insérer » qui n'a finalement pas été retenu à l'édition. Nous ignorons les causes de cette omission $^{8}$, tout en envisageant de multiples possibilités : ce texte n'aurait-il été qu'une alternative au premier "Prière d'insérer » (f1r du même tapuscrit), le choix ayant été laissé à l'éditeur? M. Dib, aurait-il changé d'avis et l'aurait-il abandonné lui-même au profit du premier « Prière d'insérer » que nous retrouvons, dans sa version développée, à la quatrième de couverture de l'édition originale ${ }^{9}$ ? C'est peu probable, étant donné l'absence de hiérarchie ou de lien quelconque entre les deux faces de ce f1. Tout ce qui les rapproche, c'est le titre qui indique leur rôle dans le recueil et qui en fait une sorte d'invitation à la réflexion du lecteur ${ }^{10}$.

6 Ce «Prière d'insérer » inédit, tel que nous le présentons ici, est le seul état connu de ce texte. Il semble assez avancé ; dans l'archive déposée actuellement à la BnF et accessible aux chercheurs, nous ne trouvons pas de tapuscrits antérieurs corrigés ni d'états manuscrits. Ceci semble inhabituel pour les chercheurs connaissant les usages d'écriture de M. Dib, qui retapait à la machine les textes qu'il avait tout d'abord rédigés, corrigés et mis au propre à la main. Comme l'archive du recueil semble assez lacunaire (absence des états manuscrits de la plupart des poèmes ; ceux qui ont pu être retrouvés semblent, vu la petite quantité de corrections, des états rédactionnels assez tardifs), nous pouvons avancer l'hypothèse selon laquelle les états antérieurs de ce tapuscrit ont partagé le sort d'autres avant-textes manquants du recueil et soit ils ont été perdus, soit ils se trouvent encore au domicile de l'auteur dans une masse de notes toujours en possession des ayant droits ${ }^{11}$.

7 Toujours est-il que ce «Prière d'insérer » repéré dans le tapuscrit le plus avancé de Formulaires est resté enfoui dans les archives de l'écrivain. C'est bien dommage car, en un certain sens, il s'agit d'un texte particulier. En effet, il est plutôt rare qu'un écrivain aussi $\operatorname{discret}^{12}$ que $\mathrm{M}$. Dib laisse entrevoir son atelier, ne serait-ce que de manière un peu conventionnelle.

C'est un court récit d'inspiration, certes, conforme au topos du poète - créateur mu par le souffle du génie ( « les pouvoirs accourent $\left.{ }^{13} »\right)$-, mais, comme toujours chez M. Dib, l'idée reçue d'inspiration ex nihilo se trouve rapidement dépassée par le principe du travail acharné et de l'exigence littéraire. Dans le cas de Formulaires, il s'agit d'une exigence d'intelligibilité. Tel est le fil conducteur de ce "Prière d'insérer ». Il semble pourtant discutable à la lumière des avant-textes de Formulaires qui révèlent un grand travail de brouillage et de défiguration du sens premier. Compte tenu de ce qui a été dit dans les lignes précédentes sur le rêve, nous sommes tentée de dire: travail d'onirisation des poèmes réunis dans Formulaires. Il n'en reste pas moins vrai que nous ignorons toujours si d'autres poèmes ont été exclus du recueil et quel était leur degré d'onirisation. Faute d'une documentation qui permettrait de trancher sur ce sujet, il ne nous reste qu'à nous fier encore une fois aux paroles de M. Dib lui-même. Dans une interview donnée treize ans après la parution du recueil, il a résumé son travail de poète ainsi : 
Tout ce qui est poème en vers est écrit d'abord spontanément sous forme de poèmes qui peuvent être publiés tels quels; mais je cherche en eux le vrai poème. Du point de vue critique ils me satisfont ces poèmes; mais du point de vue de l'exigence - une exigence indéfinissable mais sûre, intime mais sûre - dans ce que ce n'est pas exactement le poème qu'il faut, dans ce poème je le trouve, et je le sors du poème ${ }^{14}$.

La réponse à la question sur le travail d'onirisation des poèmes réunis dans Formulaires se trouve peut-être là...

\section{Fig. 1 Tapuscrit du deuxième "Prière d'insérer »}

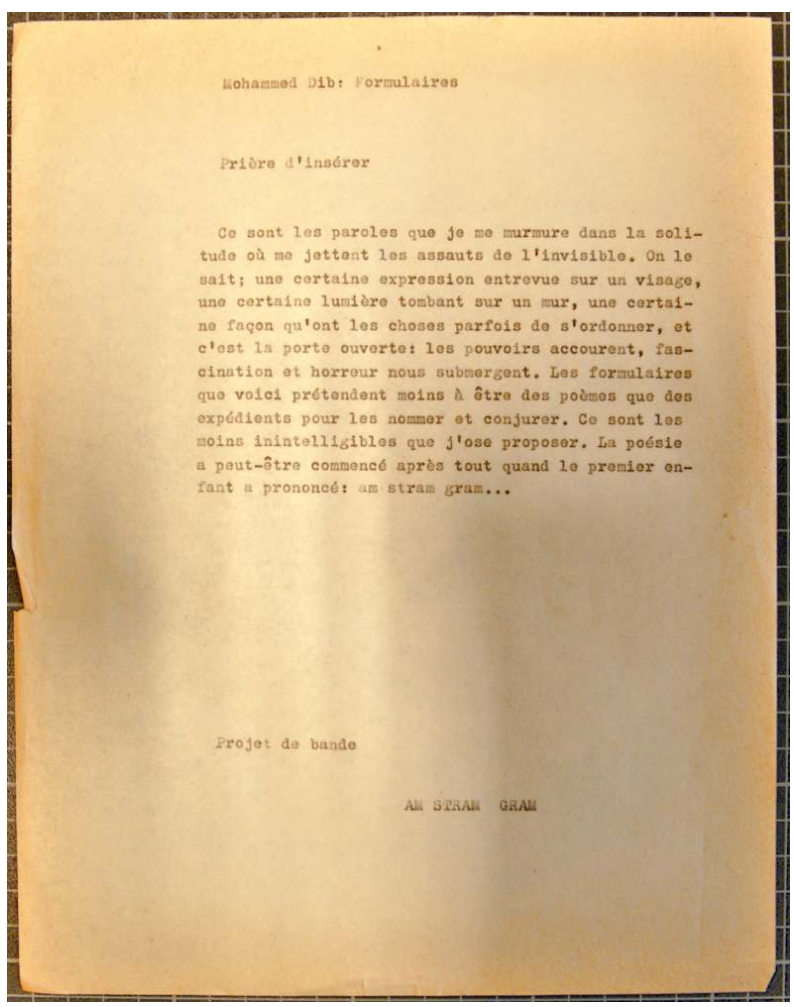

Fonds Mohammed Dib, cote NAF 28679, boîte 40 : «Formulaires », tps 5, f1 v.

Reproduction avec l'aimable autorisation de Mme Collette Dib, l'ayant droit de M. Dib

$$
* * *
$$

Comparaison entre le texte de l'incantation scandinave en norois (à gauche) et le texte de la comptine française (à droite). Le texte norois d'après Jean-Pierre Joly, " "Am stram gram..." La chevauchée des chamans ", Histoire, no 305, janvier 2006, p. 63.

\begin{tabular}{|l|l|}
\hline Emstrang Gram, & Am stram gram \\
Bigà bigà ic calle Gram & Pic et pic et colegram \\
Bure bure ic raede tan & Bour et bour et ratatam \\
Emstrang Gram & Am stram gram \\
Mos! & \\
\hline
\end{tabular}




\section{NOTES}

1. Frankétienne, propos recueillis le 20 mai 2011, pendant une conférence de l'écrivain donnée dans le cadre d'une séance de clôture du séminaire « Manuscrit francophone » (année 2010-2011) organisé par l'ITEM.

2. Fonds Mohammed Dib, cote NAF 28679, boîte 40 : « Formulaires », tps 5, f1v.

3. Selon J.-P. Joly, la comptine « Am stram gram » a dû apparaître avant le IX ${ }^{\mathrm{ème}}$ siècle (époque où l'Église catholique a déclaré une guerre ouverte aux résidus des pratiques chamaniques en France ; cf. l'instruction donnée en 852 par Hincmar, archevêque de Reims, aux prêtres qui se trouveraient confrontés aux rituels chamaniques de l'enterrement). La comptine, qui est une fidèle reprise phonétique de l'ancienne incantation chamanique de Scandinavie chantée en norois (langue commune de tous les Scandinaves utilisée au Moyen-Âge), était déjà à l'époque tellement ancrée et évidente qu'elle n'a pas éveillé les soupçons des inquisiteurs. Voir, à ce sujet, l'article de Jean-Pierre Joly " "Am stram gram..." La chevauchée des chamans », Histoire, no 305, janvier 2006, p. 60-63.

Comme la rédaction et la publication de Formulaires est décidément antérieure aux séjours de l'écrivain en Finlande (à partir de 1975), nous pouvons avancer qu'au moment de la rédaction du «Prière d'insérer » inédit, M. Dib ignorait l'origine scandinave de la comptine et qu'il s'en est servi essentiellement à cause de sa musicalité et de son inintelligibilité.

4. Dans sa recension de La Danse du Roi, «Littérature maghrébine », La Quinzaine littéraire du 15 au 30 avril 1968.

5. Dib, Mohammed, «Prière d'insérer » inédit, recueil Formulaires, tps no 5, f1v.

6. Le Petit Robert, édition 2010, version électronique sous la direction de M. Durand. Le Grand dictionnaire des synonymes et contraires sous la direction d'Y. Garnier propose même "rêver " comme un synonyme d'« imaginer » (Larousse, 2004, p. 592).

7. Dib, Mohammed « Paroles du rêve et paroles du poème (Suggestions pour l'interprétation de la poésie actuelle)», Cahiers du Collège poétique de Menton 1964, Centre culturel international de Menton, 1966, p. 92.

8. Nous avions espéré trouver la réponse à cette question dans les archives du Seuil, où se trouve la correspondance de M. Dib avec l'éditeur. Cependant, nous n'avons pu consulter le fonds avant la parution de cet article.

9. Dib, Mohammed, Formulaires, Paris, Le Seuil, 1970, $110 \mathrm{p}$.

10. M. Dib accompagnait volontiers ses œuvres de courts péritextes: les recueils Formulaires, Omnéros, Feu beau feu, L'Enfant-jazz, La Jazzoteuse en possèdent un. À cela s'ajoute un nombre important de productions épitextuelles, telles les interviews où il prend position sur son écriture. 11. A. Dib, la fille aînée de l'écrivain, confirme l'existence d'un ensemble de notes qui n'a pas encore été donné à la BnF ni mis à la disposition des chercheurs.

12. "Je ne parlerais pas de modestie mais de discrétion. Mon père était conscient de son génie mais n'en tirait pas d'orgueil personnel. »-Catherine Dib, fille de l'écrivain. Ce propos est un extrait de sa communication prononcée le 23 mai 2015 au Palais de la Culture de Tlemcen.

La pertinence de ce propos est confirmée par le témoignage de M. Dib qui, questionné par S. Jay sur le mot qui le désignerait lui-même, répondit : "Je crois que c'est "secret" mais un secret en pleine lumière... Pas celui qui se dérobe, mais celui qui s'expose. » (Salim Jay, « La Grande maison de l'écriture. Entretien avec Mohammed Dib», Horizons maghrébins, no 37-38, 1999, p. 62. Extraits de l'entretien réalisé par S. Jay pour France-Culture et diffusé en mai 1997).

13. Dib, Mohammed, « Prière d'insérer » inédit de Formulaires, f1v, tps 5. 
14. Dib, Mohammed, pour la revue CELFAN no 2, 1983, p. 23.

\section{AUTEUR}

\section{KAROLINA RESZTAK}

Doctorante, chercheuse associée à l'ITEM (CNRS-ENS). 